\title{
Paraplegia
}

\section{A New Device for the Care of Acute Spinal Injuries: The Russell Extrication Device (RED)}

\author{
A. J. Cohen, MB, BS, (Hons) UNSW, ${ }^{1}$ R. G. Bosshard, IngDip (EPF), MSc, \\ IBME, IEEE, ${ }^{2}$ J. D. Yeo, AO, MB, MS, DPRM, FRACS, FACRM ${ }^{3}$ \\ ${ }^{1}$ Chief Medical Officer, Sydney SLSAA Helicopter Rescue Service, ${ }^{2}$ Spinal Research \\ Unit, ${ }^{3}$ Director, Spinal Injuries Unit, Royal North Shore Hospital, Sydney, Australia.
}

\section{Summary}

The Russell extrication device (RED) is a new product designed for use in stabilising patients with an acute spinal vertebral and/or cord injury. An analysis was made over a 1-year period of 64 patients transported utilising the RED by the Surf Life Saving Association of Australia Sydney Helicopter Rescue Service. There were three times as many males as females; and $70 \%$ of those injured were below 30 years of age. Primary 'scene' responses accounted for just under half of all transports. Cervical injuries represent $30 \%$ of requests for helicopter transfer.

In this study no patient suffered deterioration in neurological status during the entire transfer process (including application and removal of the device). The RED has advantages for use over other currently available devices.

Key words: Spinal injury; Immobilisation; Extrication; Patient transportation; Helicopter transport; Emergency Medical Services (EMS).

The Russell extrication device (RED) (Figure) was introduced into service with the Surf Life Saving Association of Australia Sydney Helicopter Rescue Service (HRS) in November 1986. This Service has been operational for 15 years, the first aeromedical helicopter service in Australia, and is part of an Australia-wide network covering every state. As such, it is the largest and longest established civil network in the Southern hemisphere. In Sydney at the time of publication, two twin-engine state-of-the-art helicopters provide 24-hour cover to the metropolitan area and to a radius of $200 \mathrm{~km}$.

This paper looks at a 12-month spectrum of spinal injuries transported by the HRS to both Sydney Spinal Units (Royal North Shore Hospital and Prince Henry Hospital).

The RED is a ribbed injection-moulded polypropylene splint which slides behind or under an injured person (Yeo et al., 1986). The torso is immobilised by 


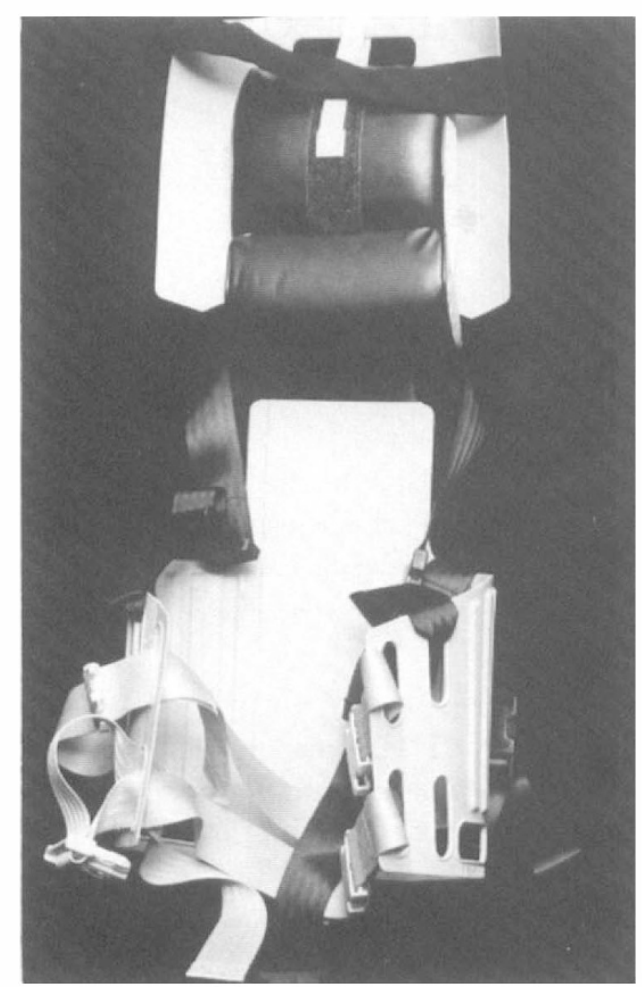

Figure The Russell extrication device (RED).

flexible wings which wrap around the thorax and abdomen. A rigid backpiece provides firm support and minimises flexion and rotation of the head. The head and neck are supported by two semicircular cushions which are placed to extend the neck. The head is further immobilised by two elastic straps fastened with Velcro to the head section of the RED. The polypropylene material is stable against ultraviolet rays, is antistatic treated, radiolucent for the purpose of X-ray examination of the spine, and is buoyant for application in water.

Two separate situations exist for the usage of the RED by the Helicopter Rescue Service doctors. Primary retrieval refers to the helicopter medical team response to the accident or incident scene. In some cases the patient may already have been prepared by ambulance personnel or bystanders and be on a spinal board or Jordan Frame, usually with a soft collar in place for cervical injuries. However, in the worst possible scenario, the patient may be unconscious, trapped in a vehicle, perhaps still in the water (fresh or salt) where the accident occurred, or be lying next to the motorbike or car from which they have been thrown, and may not have had any medical attention.

Interhospital transfer of patient from referring hospital to a major teaching hospital with a Spinal Unit is called Secondary Response. In these cases, the patient is usually already either on a spinal board, a Jordan Frame or a rigid bed, with his/her neck supported by a hard or soft collar and sandbagged in place in the case of cervical injury. In the majority of these cases, no record of the use of spinal support in the pre-hospital phase exists, or documentation is inadequate. 


\section{Methods}

A retrospective study of 64 patients with suspected or proven spinal injury transported on the RED by the Helicopter Rescue Service was undertaken.

The RED was applied by our service in a number of differing situations.

1. Where there was a proven spinal vertebral injury (with or without cord lesion) at the levels $\mathrm{Cl}$ to $\mathrm{L} 5$.

2. Where there was suspected spinal vertebral injury at these levels the RED was used.

3. Where a patient had a history of a fall or a medium to high velocity motor vehicle or motor cycle accident.

4. An acute neck flexion injury whilst diving into water.

5. A sporting injury where the patient exhibited either pain, paraesthesia, weakness or reflex abnormalities then the treating medical officer would also use the RED.

6. Any patient with a head injury and the possibility of concomitant neck injury as evidenced by mild concussion, unconsciousness or a period of lost consciousness.

In a Primary response the attending medical officer assessed the patient in situ and considered the degree of resuscitation required to stabilise the patient prior to transport. Life-threatening injuries were attended to first (i.e. airway securing and maintenance, haemorrhage and hypovolaemia).

For injuries to the cervical region, a cervical collar is positioned. The RED may be slid behind or under an entrapped patient at this stage, with the medical officer in control of the head and neck preventing any movement which contributed to the initial injury. If the patient is in a sitting position or where access is restricted, the additional straps are placed around each thigh and secured to the RED, and the abdominal straps positioned and tightened. Then the head straps are applied as below.

Alternatively, a controlled lift of the supine patient may be undertaken after appropriate and thorough briefing of the lifting team and again with a medical officer in control of the head and neck position. Once the patient is placed on the RED, the abdominal straps are first lightly fastened, and then the head straps applied. Using one hand to immobilise the patient's head, the medical officer first anchors one end of the Velcro strip and then stretches the strap across the patient's head and anchors the other end. Alternatively, with a second person controlling head movement, the medical officer can place the middle of the strap onto the patient's forehead and can then secure both Velcro end strips simultaneously. The second head strap is then applied 'crisscross' fashion across the first strap, or may be placed under the patient's chin, provided the airway is not compromised. Abdominal straps are now firmly tightened.

In the Secondary response mode, with a hospitalised patient, a full history and physical examination is first undertaken prior to consideration for transport. Other injuries are assessed, and if necessary, treated, a naso-gastric tube placed, and an indwelling urethral catheter inserted. 
Relevant X-rays and/or computerised tomography (CT) scans are also viewed. The degree of stability of the spine is assessed, the neurological damage appraised and the decision made to either reduce a fracture/disocation initially (by closed or open methods) or transport the patient. For cervical injuries an appropriate collar of the correct size is positioned and skeletal traction or sandbags removed. The medical officer then briefs the lifting team of at least three other persons of suitable strength. He positions the head and neck of the patient during the lift and the patient is raised from the bed in a controlled manner. The RED is slid under the patient who is then gently lifted downwards.

The medical officer checks the placement of the RED whilst maintaining head and neck position. The abdominal straps are connected and the head straps applied as described previously. The abdominal straps are tightened and a second, controlled lift can now be made: the patient in the RED is positioned on the transport stretcher before being loaded into the helicopter. The thigh straps are not used in this situation.

At the accepting hospital the helicopter doctor briefs the emergency unit and/or spinal team and further treatment of associated injuries is evaluated. Additional $\mathrm{X}$-ray examination may be undertaken at this stage, and even computerised tomography or magnetic resonance imaging performed with the RED in place, or it may be removed, depending on the practice of the Unit. The RED is removed in essentially a reverse of the application steps. Once the patient is free from the device, with a doctor maintaining head and neck position, skeletal traction may be applied or head and neck sandbagged in place for a cervical injury.

\section{Results}

Forty seven spinal injured patients were transported using the RED. Of 64 patients, 26 were Primary (scene) responses and 38 were Secondary transports.

Table I Spinal injuries transported on RED

\begin{tabular}{lr}
\hline $10-20$ & 19 \\
$21-30$ & 20 \\
$31-40$ & 9 \\
$41-50$ & 5 \\
$51-60$ & 5 \\
$61-70$ & 3 \\
$71-80$ & 3 \\
Total & 64 \\
\hline
\end{tabular}

Forty eight males were transported compared with 16 females. Table I shows the distribution of age groups of patients transported during the study. Table II correlates the level of vertebral injury with the degree of neurological dysfunction.

The spinal injuries occurred in a variety of differing and often bizarre circumstances. Table III outlines the major categories of injury whilst Table IV details specific modes of injury not included in a major category. 
Table II Correlation of the level of vertebral injury with the degree of neurological dysfunction

\begin{tabular}{ccccc}
\hline \multirow{2}{*}{$\begin{array}{c}\text { Vertebral level of injury -v- outcome } \\
\text { level }\end{array}$} & No & Nil loss & Incomplete & Complete \\
\hline C1-2 & 7 & 6 & 1 & \\
$2-3$ & 1 & 1 & & \\
$3-4$ & 1 & & & 1 \\
$4-5$ & 3 & 2 & 1 & 3 \\
$5-6$ & 8 & 3 & 2 & \\
$6-7$ & 3 & 2 & 1 & \\
C7-T1 & 1 & & 1 & \\
T1-2 & 0 & & & \\
$2-3$ & 0 & & & 1 \\
$3-4$ & 2 & & 1 & 1 \\
$4-5$ & 1 & & & \\
$5-6$ & 0 & & & \\
$6-7$ & 0 & & & 1 \\
$7-8$ & 1 & & & \\
$8-9$ & 1 & 1 & & \\
$9-10$ & 0 & & 1 & \\
$10-11$ & 2 & 1 & & \\
$11-12$ & 0 & & & \\
T12-L1 & 3 & 2 & 1 & \\
L1-2 & 3 & 1 & 2 & \\
$2-3$ & 6 & 2 & 3 & 1 \\
$3-4$ & 3 & 1 & 1 & 1 \\
$4-5$ & 1 & 1 & & \\
L5-S1 & 0 & & & \\
Total & 47 & & & \\
Nil vertebral/cord injury & 17 & & & \\
Soft tissue injury only & 17 & & & \\
\hline & & & & \\
& & &
\end{tabular}

Table III Major mechanisms of injury

\begin{tabular}{lr}
\hline MVA & 21 \\
MBA & 3 \\
Fall & 16 \\
Surf 'dump' & 7 \\
Other & 17 \\
Total & 64 \\
\hline
\end{tabular}

Table IV 'Other'/minor mechanisms of injury

\begin{tabular}{lr}
\hline Waterskiing & 3 \\
Rugby league/union & 3 \\
Diving into water & 2 \\
Rh. arthritis & 1 \\
Plane crash & 1 \\
Mine collapse & 1 \\
Pallets on head & 1 \\
Trotting & 1 \\
Horse riding & 1 \\
Hit on neck & 1 \\
Pyramid race & 1 \\
Steel girder crush & 1 \\
Total & 17 \\
\hline
\end{tabular}


During the period of study no patient suffered a deterioration in neurological status during the assessment, application, transfer and/or removal phase of RED usage.

Analysis of the data reveals that just over half of the patients were 'scene' responses. Males predominated over females in the ratio of 3:1 overall. The age group of the patients transported reflects a well recognised trend in acute spinal injuries noted elsewhere (Refshauge, 1987), i.e. mostly young males are affected.

\section{Discussion}

The predominance of cervical vertebral injuries transported by the helicopter service reflects the emphasis placed on this part of the spine by the general and medical communities. As is well documented, motor vehicle and motor cycle accidents provided the majority of patients for the Spinal Unit. The relatively large number of patients who suffered an injury during a fall (approximately 25\%) reflects the fact that these people often experienced their accident in inaccessible locations were helicopter extrication is required. The minor categories of spinal injury mechanisms, as is demonstrated in Table IV, once again exemplifies the broad spectrum of events which may lead to a spinal vertebral and/or cord injury.

From the finding that no patient suffered any demonstrable neurological loss during the entire procedure of assessment and treatment using the RED and subsequent helicopter transfer, it can be concluded that this represents an efficient and very effective way of treating patients with spinal injuries and will hopefully lead to further recognition and practice of this entire process.

In addition to the RED, the helicopter carries a vacuum mattress, a variety of sizes of soft cervical collars and rigid polypropylene extrication collars, a golding aluminium stretcher with foam mattress and a two piece rigid plastic stretcher with enclosed sides and rubberised mat which can be winched into the helicopter from heights as great as $35 \mathrm{~m}$ using the rescue helicopter's electric hoist.

The RED has the following advantages over the other commonly used spinal immobilisation devices. The injection moulded polypropylene composition allows undistorted radiological examination, and facilitates cleaning. The unit is easily slid behind or under a patient. The flexible wings firmly bind the unit to the patient's torso without restriction of respiration, and the head straps prevent rotation flexion and extension of the head and neck. Longitudinal movements of the RED relative to the patient are prevented by the abdominal straps. The additional application of thigh straps may be necessary when the patient is extricated from a motor vehicle.

The unit is not flammable and is antistatically treated. It is extremely light $(2 \mathrm{~kg}$ ) and of relatively low cost. The RED has the additional benefits of being compact, versatile in its application for Primary and Secondary retrieval, buoyant for use in the water, and easy to apply by a single attendant.

Stabilisation is assured for the often difficult process of patient carriage to and in the helicopter and other forms of transportation.

As a result of this and similar evaluations, the RED is already coming into wide acceptance with Ambulance services throughout Australia.

Toscano has reported that some $25 \%$ of spinal injuries occur after the initial accident or incident (i.e. during subsequent handling). The RED must surely have an important role in reducing the likelihood of further injury to the spinal cord. 


\section{Conclusion}

The Russell extrication device (RED) is a new Australian product designed for use in stabilising acute spinal vertebral and/or cord injuries and has been found to be extremely successful by the Sydney Surf Life Saving Association Helicopter Rescue Service. It has a number of advantages over similar devices which make it the preferred method of spinal immobilisation during extrication and transportation by the Service.

\section{Acknowledgement}

Equipment donated by Millar, Mitchell \& Co., PO Box 700, Double Bay NSW 2028, Australia.

\section{References}

ReFshauge W (ed) 1987, Towards the Prevention of Spinal Cord Injury. Menzies Foundation Technical Report. No. 1, p 80

TOSCANO J, A study to identify risk factors in the aetiology and cause of traumatic spinal cord paralysis. Thesis for Doctorate of Medicine, University of Melbourne.

YeO JD, Bossard RD, MILlaR GK 1986, A fully molded extrication device for the spinal injured. Paper presented in the proceedings of the International Medical Society of Paraplegia, Scientific Meeting, Oslo, Norway. 